\title{
HELIOSEISMOLOGY OF A REALISTIC MAGNETOCONVECTIVE SUNSPOT SIMULATION
}

\author{
D. C. Braun ${ }^{1}$, A. C. Birch ${ }^{1}$, M. Rempel ${ }^{2}$, and T. L. Duvall, Jr. ${ }^{3}$ \\ ${ }^{1}$ Northwest Research Associates, 3380 Mitchell Lane, Boulder, CO 80301, USA; dbraun@cora.nwra.com, aaronb@cora.nwra.com \\ 2 National Center for Atmospheric Research, HAO Division, 3080 Center Green Drive, Boulder, CO 80301, USA; renpel@ucar.edu \\ ${ }^{3}$ Solar Physics Laboratory, NASA Goddard Space Flight Center, Greenbelt, MD 20771, USA; Thomas.L.Duvall@nasa.gov \\ Received 2011 September 15; accepted 2011 November 11; published 2011 December 14
}

\begin{abstract}
We compare helioseismic travel-time shifts measured from a realistic magnetoconvective sunspot simulation using both helioseismic holography and time-distance helioseismology, and measured from real sunspots observed with the Helioseismic and Magnetic Imager instrument on board the Solar Dynamics Observatory and the Michelson Doppler Imager instrument on board the Solar and Heliospheric Observatory. We find remarkable similarities in the travel-time shifts measured between the methodologies applied and between the simulated and real sunspots. Forward modeling of the travel-time shifts using either Born or ray approximation kernels and the sound-speed perturbations present in the simulation indicates major disagreements with the measured travel-time shifts. These findings do not substantially change with the application of a correction for the reduction of wave amplitudes in the simulated and real sunspots. Overall, our findings demonstrate the need for new methods for inferring the subsurface structure of sunspots through helioseismic inversions.
\end{abstract}

Key words: Sun: helioseismology - Sun: interior

\section{INTRODUCTION}

The goal of local helioseismology is the assessment and modeling of the properties of solar acoustic ( $p$ mode) and surface gravity ( $f$ mode) waves to determine mass flows and wave-speed variations within local volumes in the solar interior. Sunspots have been the subject of local helioseismic study for more than two decades (e.g., Bogdan \& Braun 1995), yet considerable uncertainty exists in the measurement, interpretation, and modeling of helioseismic signals around the strong magnetic fields present in spots (e.g., see the review by Gizon et al. 2010).

The first inferences of the sound-speed structure beneath sunspots were made from forward modeling of the phase shifts detected between waves propagating into and out of sunspots as measured through Hankel analysis (Braun 1995; Fan et al. 1995). The best model, which reproduced the general properties of the observed phase shifts, consisted of a shallow (approximately $1 \mathrm{Mm}$ deep) region of faster wave speed immediately beneath sunspots (Fan et al. 1995). The subsurface three-dimensional (3D) wave-speed structure of sunspots was subsequently deduced from inversions (e.g., Kosovichev 1996; Kosovichev \& Duvall 1997; Kosovichev et al. 2000) of $p$-mode travel-time shifts, defined as travel-time differences from those expected from measurements of travel times in the quiet Sun, as measured using time-distance helioseismology. These shifts typically show a variation with horizontal phase speed $w$ (the temporal frequency $\omega$ divided by the horizontal wavenumber $k$ ), ranging from positive (longer travel times) at small phase speeds to negative (shorter travel times) at larger phase speeds. Deeper penetrating modes have increasing phase speed, so the traveltime variation with $w$ provides the basis for two-layer wavespeed models (e.g., Kosovichev et al. 2000; Couvidat et al. 2005) which extend downward to approximately $10 \mathrm{Mm}$ below the surface. Structural inversions of the wave-speed perturbations underneath active regions have also been made with ring-diagram analysis (e.g., Basu et al. 2004; Gizon et al. 2009; Kosovichev et al. 2011). Some qualitative agreement between results from ring-diagram and time-distance analyses has been suggested (Basu et al. 2004; Kosovichev et al. 2011), although it is clear that substantial systematic quantitative differences exist between ring-diagram, time-distance, and Hankel analyses of sunspots (Gizon et al. 2009, 2010).

Complicating the interpretation of helioseismic inferences in sunspots are observations and analyses which may show evidence of strong surface (or near-surface) contributions to the helioseismic signatures associated with active regions (Lindsey \& Braun 2005; Schunker et al. 2005; Korzennik 2006; Braun \& Birch 2006; Couvidat \& Rajaguru 2007). Observations which remain unaccounted for in most helioseismic models of sunspots include the variation of travel times (or phase shifts) with the line of sight (Schunker et al. 2005, 2007, 2008; Zhao \& Kosovichev 2006) and the type of filtering applied to the data (Braun \& Birch 2008; Thompson \& Zharkov 2008; Gizon et al. 2009; Zhao et al. 2010). Travel-time shifts within sunspots also show strong variations, at fixed phase speed, with frequency. These variations include changes of sign (Braun \& Birch 2006, 2008; Couvidat \& Rajaguru 2007). Similar variations and sign changes have been qualitatively reproduced with artificial data generated from numerical simulations including both hydrostatic models (Birch et al. 2009) and magnetostatic models (Moradi et al. 2009) in which no slower layer is present.

Additional evidence for shallow models is provided by numerical forward modeling of the wave propagation through sunspot-like magnetic fields which includes the effects of mode conversion (Crouch et al. 2005; Cameron et al. 2011). These models are consistent with shallow, positive wave-speed perturbations below sunspots (as reviewed by Moradi et al. 2010). The impact of possible surface effects and the sensitivity of the measurements to methodology (e.g., filtering) on helioseismic models and the interpretation of observations in the vicinity of sunspots remains unknown. Arguments have been made that many of these effects should not qualitatively change the inferences of traditional time-distance inversions (e.g., Zhao \& Kosovichev 2006; Zhao et al. 2010; Kosovichev 2010). Some preliminary tests with laterally homogeneous magnetostatic models (Crouch et al. 2011) suggest that inversions for 
sound-speed perturbations in magnetic fields typical of sunspot umbra are only reliable when the sensitivity kernels employed in the inversions explicitly include the physical effects of the magnetic field. In particular, the treatment of the influence of the magnetic field of the sunspot on the waves as being equivalent to a local change in the isotropic wave speed is not accurate in these models (Crouch et al. 2011).

The generation of artificial data through numerical modeling can provide the means by which a number of these issues may be explored. Over the last decade a large variety of numerical simulations of wave propagation in the context of helioseismology have been studied (e.g., Birch et al. 2001, 2011; Jensen et al. 2003; Tong et al. 2003; Mansour et al. 2004; Benson et al. 2006; Hanasoge et al. 2006; Khomenko \& Collados 2006; Shelyag et al. 2006, 2007; Parchevsky \& Kosovichev 2007, 2009; Zhao et al. 2007; Braun et al. 2007; Cameron et al. 2008, 2011; Crouch et al. 2011). Recent 3D radiative magnetoconvective simulations of realistic sunspot-like structures have been performed (Rempel et al. 2009b; Rempel 2010) and specific data sets appropriate for the application of helioseismic methods are publicly available. ${ }^{4}$ In this paper, our overriding goal is to test some assumptions which form the basis for traditional 3D structural inversion methods for sunspots. To do this we apply forward modeling to helioseismic measurements performed using the vertical velocity sampled at a constant optical depth near the surface of a realistic sunspot-like structure. We use the term "traditional" here to mean methods which are designed to probe isotropic wave-speed perturbations. The applicability of our findings to the modeling of the subsurface properties of real sunspots is to a large extent determined by the resemblance of the measurements to those made on the Sun. An important subgoal of this work is therefore to compare the travel-time shifts observed from the MHD simulations with those observed in two sunspots. In addition, we compare the travel-time shifts obtained using two procedures, helioseismic holography and time-distance helioseismology.

\section{DATA}

\subsection{Synthetic Data from Convective MHD Simulations}

The numerical sunspot models utilized here are based on the methods presented by Rempel et al. (2009a, 2009b) and differ mostly in initial setup and domain size, as well as resolution. We present here a simulation of a single sunspot in a domain with the dimensions $49.152 \times 49.152 \times 8.192 \mathrm{Mm}^{3}$ with a resolution of $96 \times 96 \times 32 \mathrm{~km}^{3}$, resulting in a grid size of $512 \times$ $512 \times 256$. Boundary conditions are periodic in the horizontal directions. The bottom boundary is open for convective flows and the magnetic field is vertical. In regions with more than $2.5 \mathrm{kG}$ field strength the bottom boundary is closed to prevent a fast decay of the sunspot on a timescale of hours as a consequence of the rather short convective timescales in an $8 \mathrm{Mm}$ deep domain. The role of the bottom boundary for the overall stability of the sunspot and a comparison with an open boundary condition are discussed in more detail in Rempel (2011). The top boundary is closed for flows and the magnetic field is matched to a potential field extrapolation. The simulation was started from a thermally relaxed convection run in which we inserted an axisymmetric self-similar magnetic field structure with a total flux of $1.2 \times 10^{22} \mathrm{Mx}$. The initial field strength and radius at the bottom boundary are $8 \mathrm{kG}$ and $7 \mathrm{Mm}$, and the initial field

\footnotetext{
4 http:download.hao.ucar.edu/pub/rempel/sunspot_models
}

at the top boundary is $2.66 \mathrm{kG}$. We did not adjust the pressure in the initial state to account for magnetic forces but rather allow the adjustment to happen as part of the initial time evolution. To maintain manageable time steps in the numerical simulation we artificially limited Alfvén waves to $60 \mathrm{~km} \mathrm{~s}^{-1}$ in the region above the sunspot umbra where the ratio of gas pressure to magnetic pressure $\beta \ll 1$. We conducted a control experiment with a cutoff at $120 \mathrm{~km} \mathrm{~s}^{-1}$ and did not find significant differences in the resulting power spectra. The data analyzed here start from $3 \mathrm{hr}$ after initialization to about $30 \mathrm{hr}$, leading to a continuous time series of $27.3 \mathrm{hr}$. From the simulation we extracted maps of the vertical velocity at several constant optical depth $(\tau)$ and height levels for helioseismic analysis. The analysis presented in this paper is based on the $\tau=0.01$ level.

Figure 1 shows, top to bottom, the bolometric intensity, magnetogram at $\tau=1$, and the subsurface magnetic field strength on a vertical slice through the center of the sunspot. The left panels show a snapshot from the middle of the time series analyzed and the right panels show temporal averages over the full length of the time series $(27.3 \mathrm{hr})$. Due to the rather low resolution of this simulation in combination with the horizontal periodicity we did not obtain an extended penumbra in this simulation, i.e., our sunspot is a "naked" sunspot, which is similar to a pore surrounded by a ring of inflowing material. Extended penumbrae can be obtained either by placing opposite polarity flux nearby (Rempel et al. 2009a) or by artificially enhancing the inclination angle through the top boundary condition (Rempel 2010). Figure 2 shows time and azimuthal averages of the sound speed $(c)$, fast-mode speed, and density in the simulated sunspot.

\subsection{HMI and MDI Observations of Sunspots}

Two sunspots were selected for comparison with the simulated sunspot. We selected two mature spots (in active regions AR10615 and AR11092) which had umbral diameters of 23 and $31 \mathrm{Mm}$, respectively, and are comparable to the simulated spot size of $27 \mathrm{Mm}$. The data set for AR10615 consisted of 17 hr of high-resolution Dopplergrams obtained from the Michelson Doppler Imager (MDI; Scherrer et al. 1995) on board the Solar and Heliospheric Observatory (SOHO) starting on 2004 May 22, 09:48 UT. A region $74 \times 74 \mathrm{Mm}^{2}$ mapped using a Postel projection centered on the sunspot and tracked at the Snodgrass rotation rate was extracted from the high-resolution field of view for analysis. This sunspot (AR10615) was among the group of 16 sunspots studied previously by Couvidat \& Rajaguru (2007). The data set for AR11092 was obtained from the Helioseismic and Magnetic Imager (HMI; Schou et al. 2011) on board the Solar Dynamics Observatory starting on 2010 August 4, 0:00 UT. A Postel-projected and tracked datacube, $373 \times 373 \mathrm{Mm}^{2}$ and $24 \mathrm{hr}$ duration, centered on the sunspot was provided by the HMI time-distance team.

\subsection{Power Spectra}

Figure 3 shows power spectra of the synthetic vertical velocity from the MHD simulation (right panel) and a region around the sunspot in AR11092 extracted from the tracked HMI Dopplergrams. The power spectra were obtained by performing Fourier transforms (in both spatial dimensions and in time) of the data and azimuthally summing the power in the wavenumber domain in bins of constant wavenumber $k$. The power spectrum for the synthetic data agrees well with the HMI spectrum in some portions of the $k-\omega$ domain and disagrees elsewhere; 


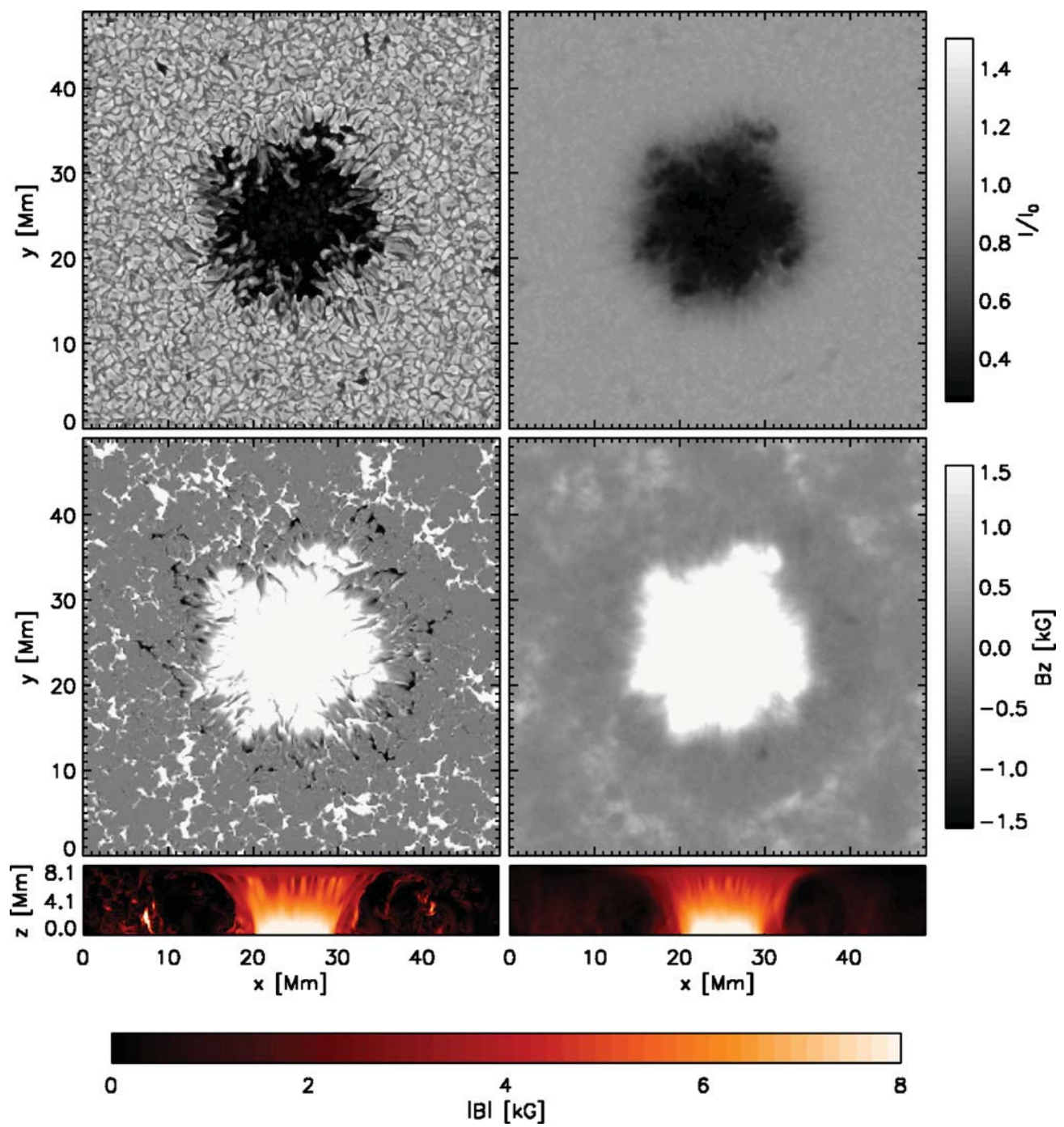

Figure 1. Bolometric intensity (top panels) and vertical magnetic field (middle panels) at $\tau=1$. The bottom panels show the subsurface magnetic field strength on a vertical slice through the center of the sunspot. The left panels show a snapshot from the middle of the time series analyzed; the right panels show temporal averages over the full length of the time series $(27.3 \mathrm{hr})$.

the largest disagreement is due to reflections of waves from the bottom boundary of the computational domain. The diagonal dashed line denotes a horizontal phase speed $w$ of $29 \mathrm{~km} \mathrm{~s}^{-1}$ which is the speed of sound at the bottom of the domain. Waves with smaller phase speeds (i.e., which fall on the right side of the dashed line) avoid this reflection and their resonance frequencies show good agreement with the solar spectra.

Phase-speed filters, which are commonly used in both time-distance helioseismology (hereafter TD) and helioseismic holography (hereafter $\mathrm{HH}$ ), can isolate waves in $k-\omega$ space where the agreement between the simulation and the Sun is acceptable. The solid and dashed black lines indicate the range in phase speed spanned by the full width at half-maxima (FWHMs) of two different Gaussian filters in phase speed. These filters, denoted TD1 and TD4, respectively, are the first and fourth of the standard phase-speed filters tabulated by Couvidat et al. (2006) in order of increasing phase speed. It is apparent that the high phase-speed edge of filter TD4 coincides with the sound speed at the bottom of the simulated domain. Figure 4 shows the resulting power spectra after applying this filter to both data sets. This filter (as do those centered on smaller phase speeds) removes most of the reflected wave contribution.

\section{TRAVEL-TIME MEASUREMENTS}

\subsection{Helioseismic Holography}

We employ a general procedure for surface-focused HH (see Braun \& Birch 2008), which is analogous to the application of center-annulus correlations in time-distance helioseismology employed later (Section 3.2). With only small differences, this procedure is nearly identical to recent $\mathrm{HH}$ analysis of synthetic data containing pure sound-speed perturbations (Birch et al. 2011). As a first step, we apply the set of phase-speed filters to the synthetic data. For each phase-speed filter there is a pupil function that is employed (see Braun \& Birch 2006, 2008). To minimize issues relating to wave reflections from the bottom of the computational domain (see Section 2.3) we use the first four phase-speed filters (TD1-TD4) described in Table 1 of Couvidat et al. (2006). The inner and outer radii of the pupils are determined by the range of annuli given in the same table. We use a full annular pupil for these measurements. Next, we measure the local control correlations using the $\mathrm{HH}$ Green's functions of Lindsey \& Braun (2000). There are two correlations for each filter used, which correspond to incoming and outgoing waves. These are analogous to center-annulus cross-covariance measurements used in time-distance methods 

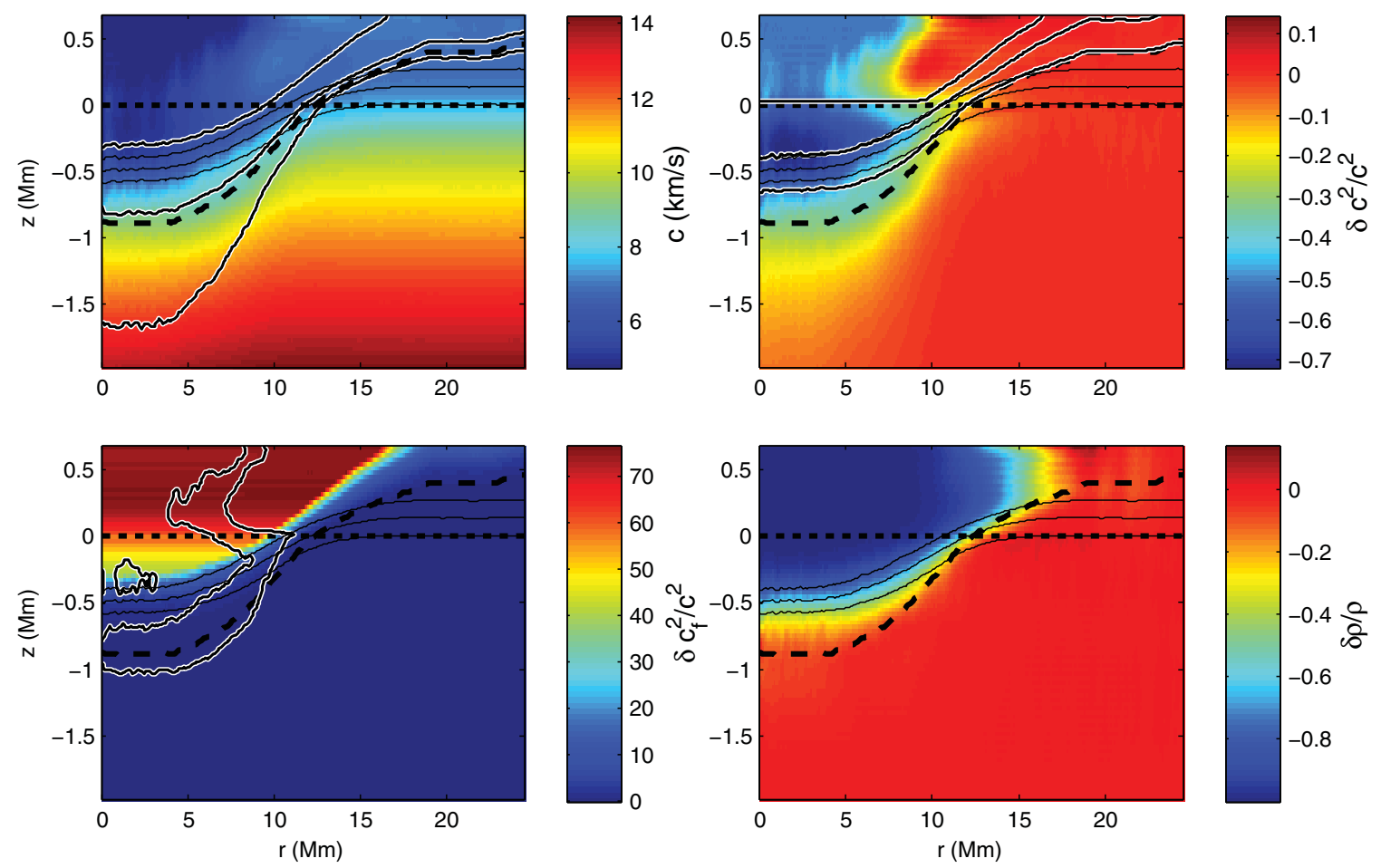

Figure 2. Time and azimuthal averages of the sound speed, fast-mode speed, and density in the simulated sunspot. In all of the panels, the horizontal black dashed line shows the height $z=0$, the heavier dashed black line shows the level where the sound speed is equal to the Alfvén speed, and the thin black lines show (from bottom to top) $\tau=1,0.1$, and 0.01 . All of the perturbations are measured relative to the values of the relevant quantities at the largest radius. The top left panel shows the sound speed (in colors) together with contours where the Alfvén speed is 5, 10, and $15 \mathrm{~km} \mathrm{~s}^{-1}$ (black lines with white borders). The top right panel shows the fractional perturbation to the square of the sound speed (colors) and contours where the fractional perturbation to the square of the fast-mode speed has values of 1,10 , and 60. The bottom left panel shows the same two variables as the top right panel, but now the fractional perturbation to the square of the fast-mode speed is shown in color and the contours show where the fractional perturbation to the square of the sound speed takes the values $-0.7,-0.45$, and -0.25 . The bottom right panel shows the fractional perturbation to the density. The vertical range covers only the top two Mm of the simulation domain.
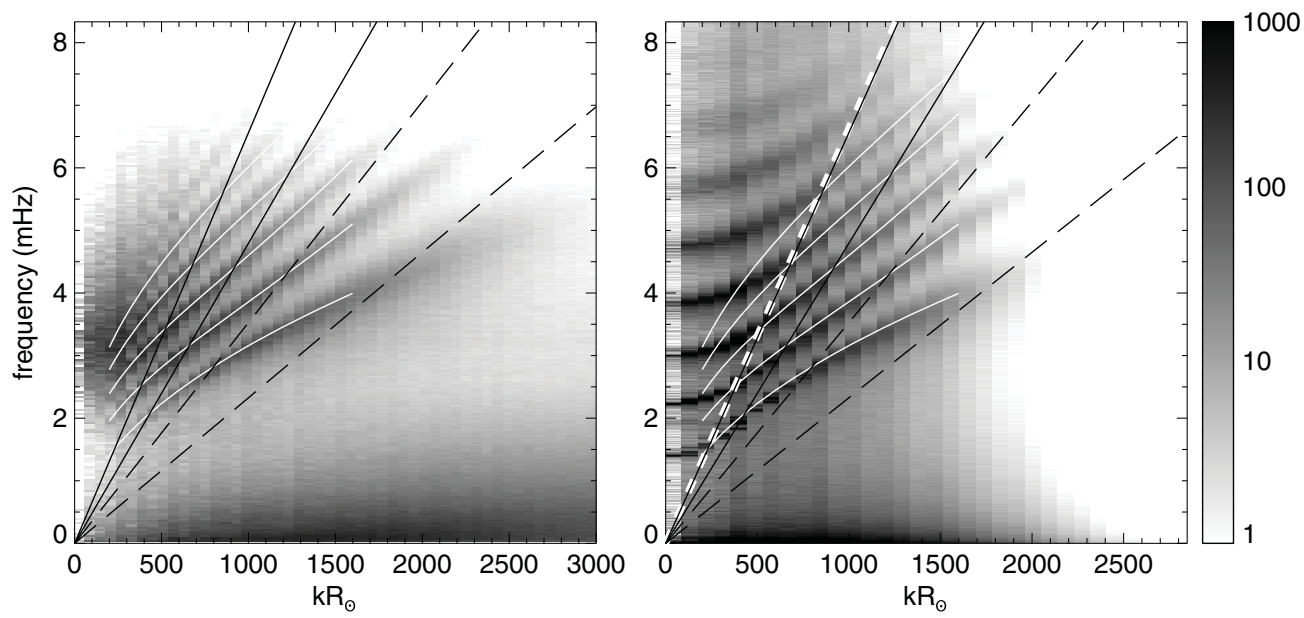

Figure 3. Left panel: power spectrum of the line-of-sight velocity obtained from HMI Dopplergrams of a region around the sunspot in AR11092 observed for a $24 \mathrm{hr}$ interval. Right panel: power spectrum of the vertical velocity at an optical depth of 0.01 from the simulation. The gray scale indicates the relative power on a logarithmic scale. The white curves show polynomial fits to tables of solar $p$-mode frequencies. The black dashed lines indicate the range in phase speed spanned by the full width at half-maxima (FWHMs) of the phase-speed filter TD1. The solid black lines indicate the range spanned by the FWHM of filter TD4. The white dashed line in the right panel indicates where the phase speed is equal to the sound speed of the bottom of the simulation. To the right of the dashed line, the ridges from the power spectra of the simulation are aligned with the solar ridges. To the left of the white dashed line, the ridges in the simulation deviate significantly from the solar ridges in a distinctive way indicative of the presence of reflection from the bottom of the computational domain.

(e.g., Gizon \& Birch 2005). These correlations are performed by a multiplication in the spatial Fourier domain of the data with the Green's functions which makes use of the horizontal spatial periodicity of the data. Next, we apply boxcar filters in the temporal frequency dimension to the correlations. We employ filters centered at 11 frequencies between $2.75 \mathrm{mHz}$ and
$5.25 \mathrm{mHz}$, equally spaced $0.25 \mathrm{mHz}$ apart and with bandpass widths equal to $0.25 \mathrm{mHz}$. Finally, we measure travel-time shifts from the filtered correlations. For this, we use the phase method described by Equations (1)-(3) of Braun \& Birch (2008) which relate the travel-time shifts to the phase of the temporal Fourier transform of the control correlations. A "quiet-Sun" 

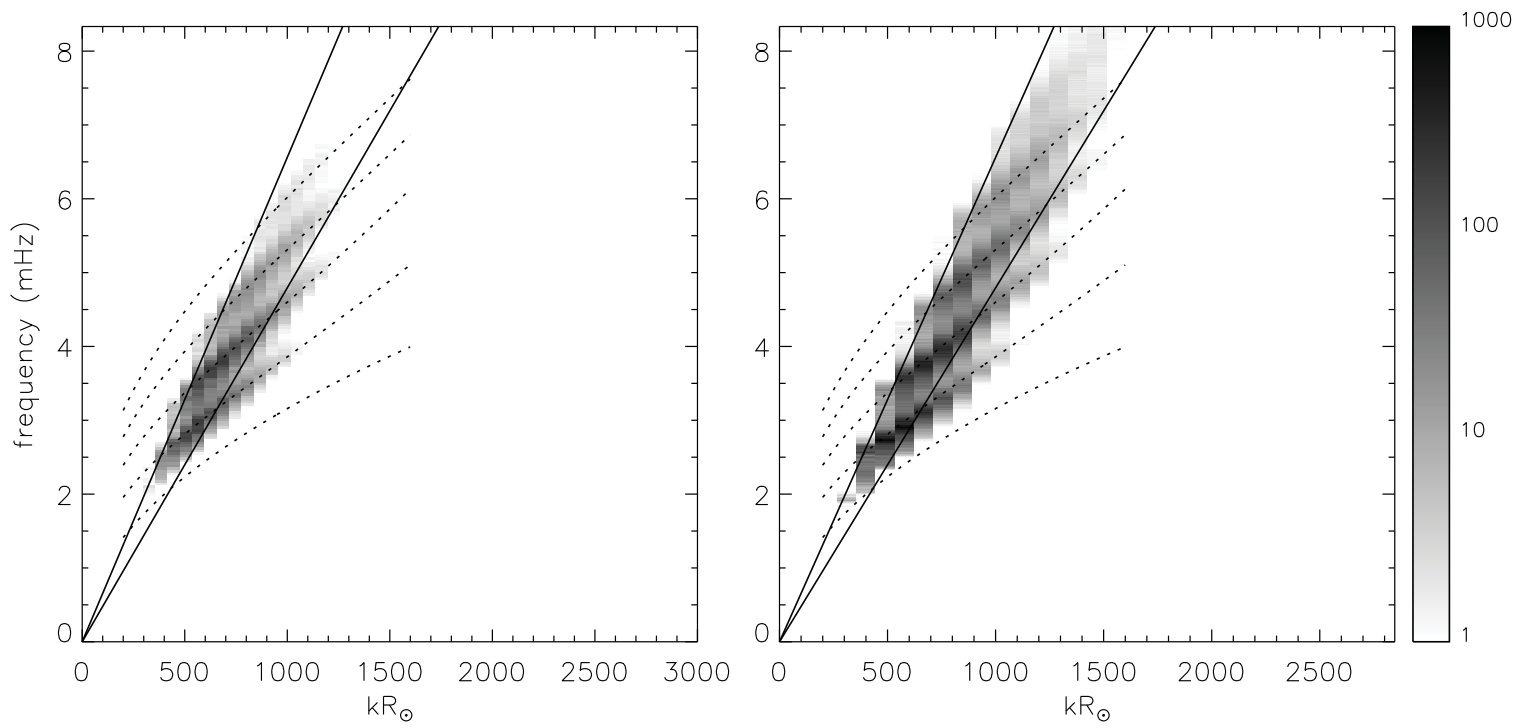

Figure 4. Same as Figure 3 but showing the power spectra of the vertical velocities after applying the phase-speed filter TD4. The filter is effective in cutting off almost all of the power to the left of the dashed line in Figure 3, such that the contribution of the reflected waves is essentially eliminated.

phase, determined from averages of the control correlations over pixels with time-averaged magnetic field strengths below $50 \mathrm{G}$, is subtracted from the observed control correlation phase. This corrects dispersion effects in the HH Green's functions.

\subsection{Time-Distance}

A standard procedure for surface-focused time-distance analysis was applied to the input datacubes (Duvall et al. 1997). This includes multiplication by phase-speed filters in the Fourier domain. The phase-speed filters used are the same as for the $\mathrm{HH}$ analysis, the first four (TD1-TD4) from Table 1 of Couvidat et al. (2006). The horizontal spatial periodicity of the data was used to extend the datacubes horizontally so that correlations and travel times could be measured to the edge of the nominal field. Center-quadrant correlations are computed for distances encompassing the range described in Table 1 of Couvidat et al. (2006). These are combined into inward and outward directions of wave travel direction.

After the phase-speed and frequency filters are applied, time-distance cross-correlations $C(\Delta, t)$ are measured for each center point, for each surrounding 1 pixel wide subannulus of radius $\Delta$, for all time lags $t$. These cross-correlations are then shifted in time (similar to the procedure described by Hindman et al. 2004) according to a reference curve $\tau(\Delta)$ and then summed over all of the subannuli that constitute a particular annulus,

$$
\overline{C_{ \pm}}(t)=\sum_{i} C\left(\Delta_{i}, t \pm \tau\left(\Delta_{i}\right)\right)
$$

where the index $i$ labels the 1 pixel wide subannuli. We then measured the ingoing and outgoing travel-time shifts from the cross-correlations $\bar{C}_{ \pm}$using a one-parameter fit (Gizon \& Birch 2002).

\subsection{Travel-time Maps}

Figures 5 and 6 show samples of the mean travel-time shifts, which represent the average of the incoming and outgoing traveltime shifts relative to quiet-Sun values, as determined from $\mathrm{HH}$ and TD, respectively. The two sets of maps are remarkably similar and show the following general features. The travel

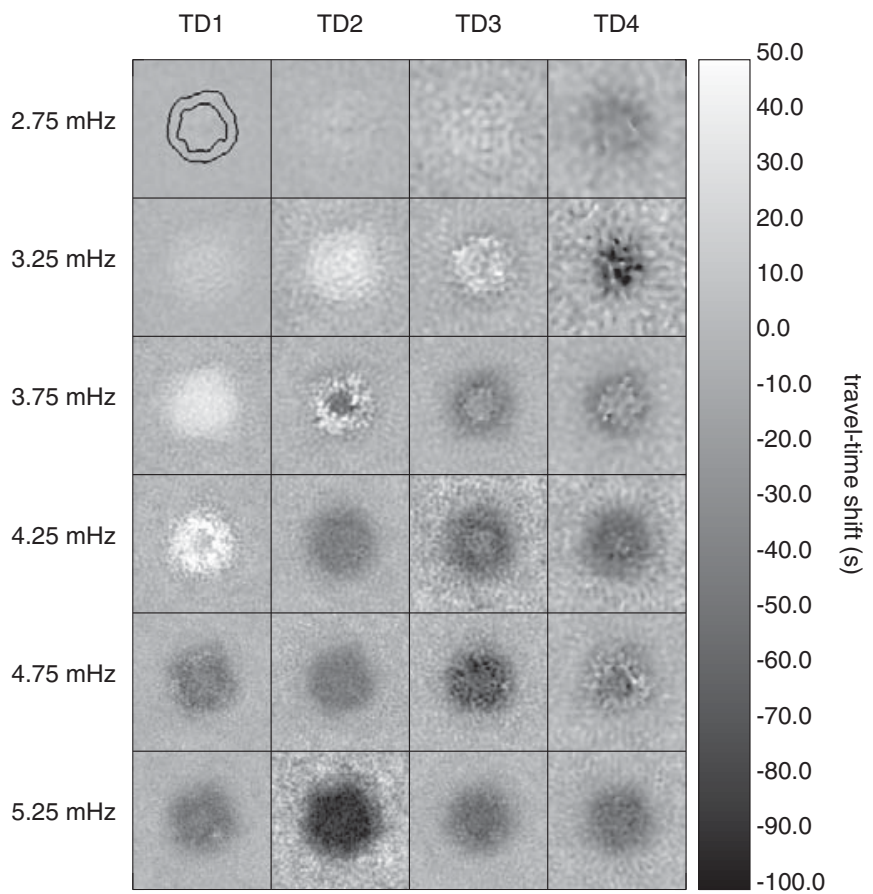

Figure 5. Mean travel-time shifts measured with helioseismic holography and using phase-speed filters in combination with frequency bandpass filters for the simulation. The vertical columns show the phase-speed filter used, increasing from $12.8 \mathrm{~km} \mathrm{~s}^{-1}$ (TD1) in the leftmost column to $24.8 \mathrm{~km} \mathrm{~s}^{-1}$ in the rightmost column. The labels on the left side illustrate the central frequency of the bandpass filter. For brevity, not all maps are shown. The contours in the upper left panel show the umbral and penumbral boundaries as defined in the text.

times in the simulated spot show a mixture of positive and negative shifts depending on the frequency and phase-speed filters used. The travel-time shifts at $2.75 \mathrm{mHz}$ are the smallest in amplitude while the travel-time shifts at frequencies above about $4.5 \mathrm{mHz}$ tend to show strong negative shifts. At intermediate frequencies, positive travel-time shifts are observed at smaller frequencies and phase speeds, while negative shifts are observed at higher frequencies and phase speeds. There are some filter combinations in which both negative and positive shifts are 


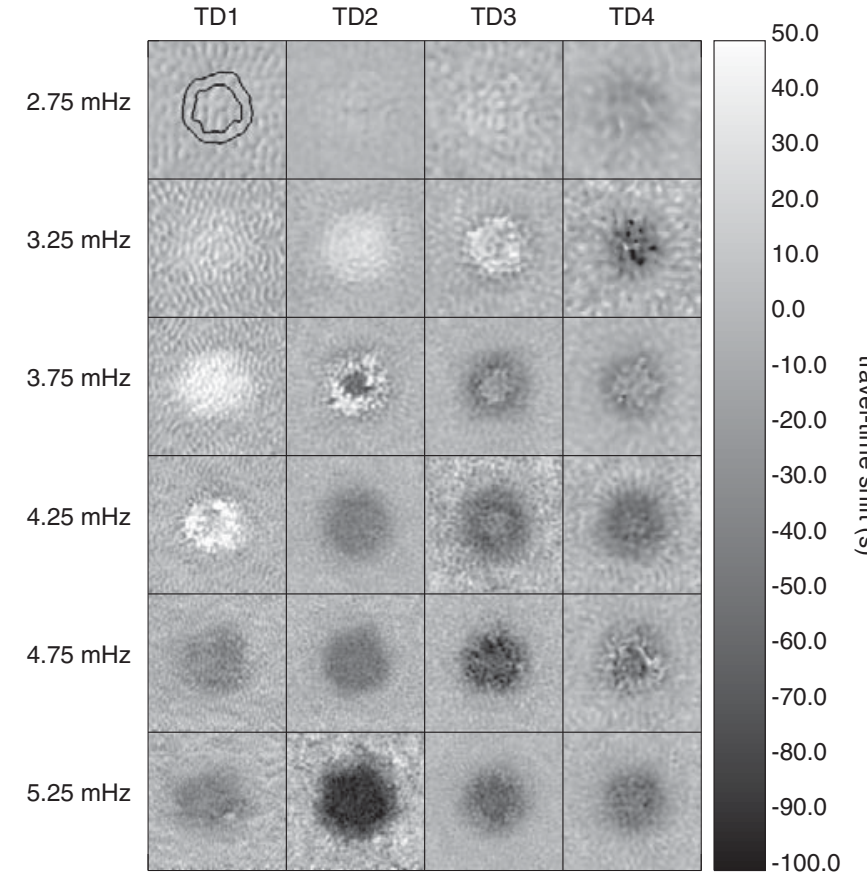

Figure 6. Mean travel-time shifts, measured with the time-distance method and using phase-speed and frequency bandpass filters for the simulation, as in Figure 5 .

observed within the spot. It is noteworthy that, for filters TD1 through TD3, the change in sign of the travel-time shift from positive to negative values with increasing frequency is reminiscent of the behavior seen in real sunspots using both HH (Braun \& Birch 2006, 2008; Gizon et al. 2009) and TD (Couvidat \& Rajaguru 2007) methods. This is confirmed by the direct comparisons with the sunspots observed with HMI and MDI discussed in the next section.

To facilitate quantitative comparisons, both between TD and $\mathrm{HH}$ methods and between the simulated and real sunspots discussed later, we average the travel-time shifts over the "umbra" and "penumbra" of the spot. The umbra is defined as the location where the continuum intensity is less than half of the quiet-Sun intensity (where the quiet Sun has been defined previously) and the penumbra is defined as between 0.5 and 0.9 times the quiet-Sun intensity. Figure 7 shows the results for both TD and HH measurements of the simulated sunspot. With few exceptions, the averaged travel-time shifts determined from TD agree within a few seconds with those determined from $\mathrm{HH}$. The largest differences appear in the results for filter TD1, where a systematic difference on the order of $10 \mathrm{~s}$ appears at the highest frequencies. An increase in noise also appears in the TD maps, especially for filter TD1. The cause of this is unknown.

The agreement in the travel-time maps made with TD and $\mathrm{HH}$ demonstrated here is remarkable in light of previous comparisons (e.g., Moradi et al. 2010). In the comparisons presented by Moradi et al. (2010), of TD and HH travel-time maps made for a sunspot observed by MDI, there was very good agreement between the methods for large phase-speed filters but evidence of systematic differences for small phasespeed filters. The reasons for the discrepancy appear to be due to the sensitivity of the travel-time measurement to the frequency content of the cross-covariance functions (e.g., see Figure 1 of Braun et al. 2011). The agreement between methodologies improves with the use of narrow temporal-frequency boxcar filters such as employed in this study.

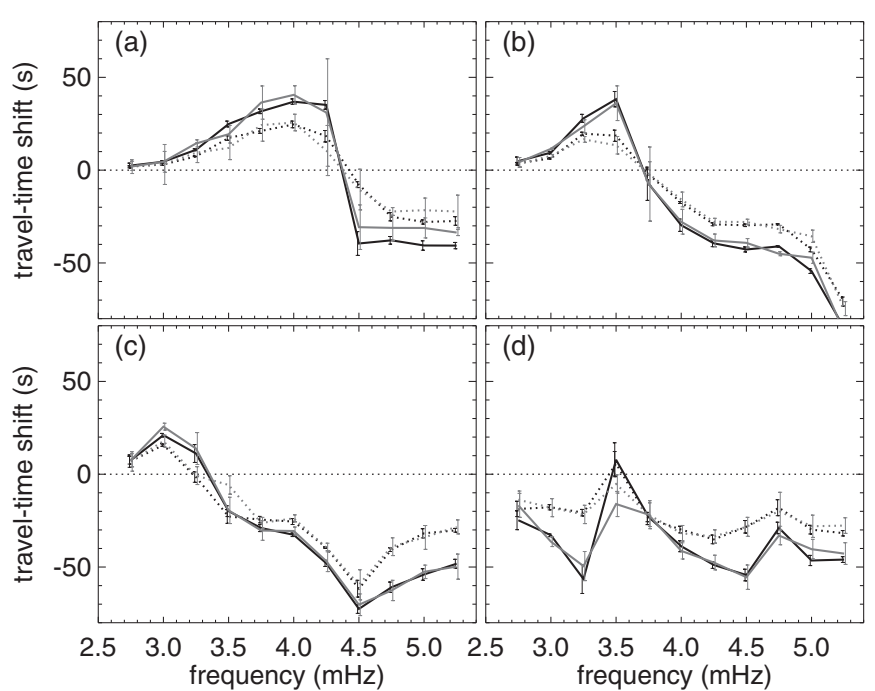

Figure 7. Averages over the umbra (solid curves) and penumbra (dotted curves) of the mean travel-time shifts for the magnetoconvective simulation as a function of the central frequency of the bandpass filters used. The black curves show the results obtained using helioseismic holography and the gray curves show the results obtained using time-distance helioseismology. The error bars represent the total spread in values obtained by measurements of portions of each umbral (or penumbral) region divided into four quadrants. For clarity, the error bars for the time-distance measurements are displaced slightly to the right of the corresponding holography measurements. Panels (a)-(d) show the results for the phase-speed filters TD1 through TD4, respectively.

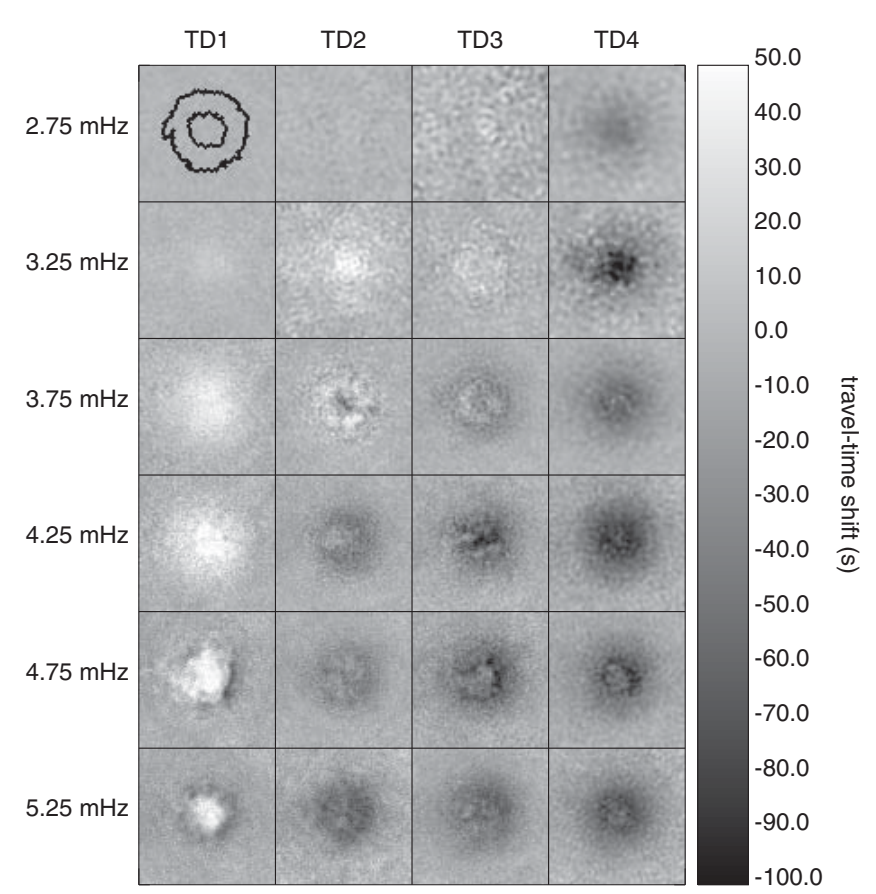

Figure 8. Mean travel-time shifts measured with helioseismic holography using phase-speed filters in combination with frequency bandpass filters for the HMI observations of the sunspot in AR11092.

\subsection{Comparisons with Sunspots}

Figure 8 shows the mean travel-time shifts measured with $\mathrm{HH}$ using phase-speed filters in combination with frequency bandpass filters for the HMI observations of the sunspot in AR11092. A comparison with Figure 5 shows many similarities with, and a few differences from, the set of travel-time shifts measured from the simulated data set. In particular, the traveltime shifts for AR11092 are predominantly positive (i.e., longer 


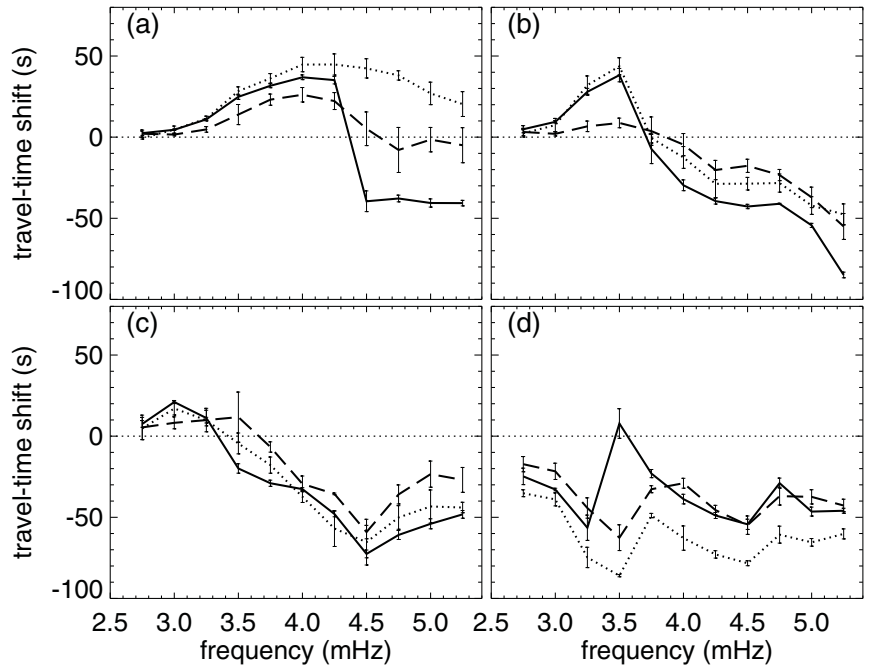

Figure 9. Averages over the umbra of the mean travel-time shifts for the magnetoconvective simulation and for two sunspots as a function of the central frequency of the bandpass filters. The solid curves show the results for the magnetoconvective simulation, the dotted curve shows the results for the sunspot in AR11092, and the dashed curve shows the sunspot in AR10615. The error bars represent the total spread in values obtained by measurements of portions of each umbral region divided into four quadrants. Panels (a)-(d) show the results for the phase-speed filters TD1 through TD4, respectively.

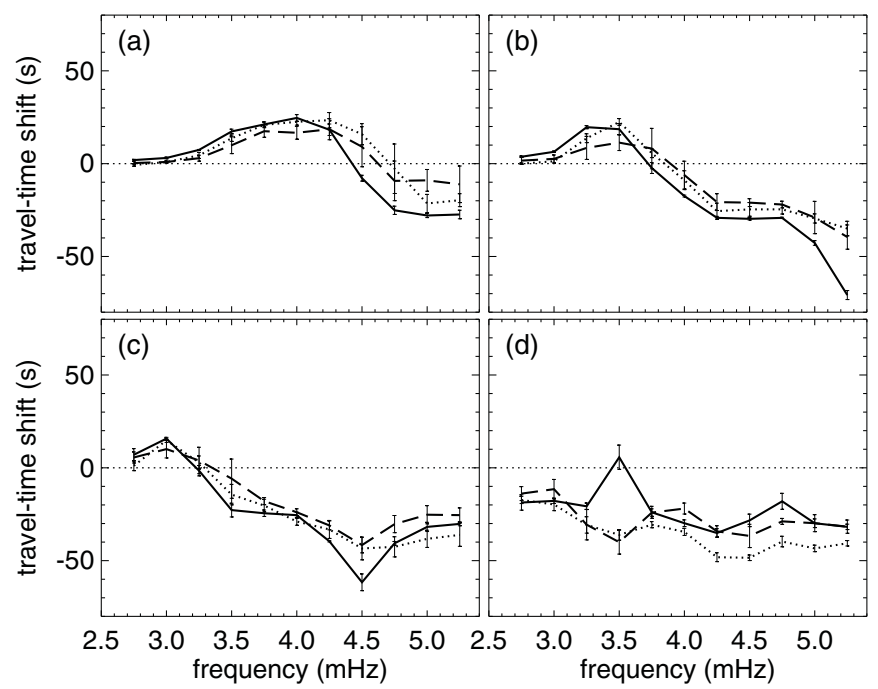

Figure 10. Averages over the penumbra of the mean travel-time shifts for the simulation and the two sunspots. The lines have the same meaning as for Figure 9.

travel times) at lower temporal frequencies and phase speeds and negative (shorter travel times) at higher frequencies and phase speeds, which are trends also observed in the simulated sunspot measurements. A noticeable difference is that, at frequencies above $4.5 \mathrm{mHz}$, the travel-time shifts in the center of the sunspot are positive while the corresponding shifts in the simulated spot are negative.

Figures 9 and 10 show quantitative comparisons of the umbral and penumbral averages, respectively, between the $\mathrm{HH}$ measurements made for the simulated spot and the two real sunspots. There is good qualitative agreement between the travel-time shifts of the simulated sunspot and those of the two real spots. There is also remarkable quantitative agreement between the simulated and real spots for most of the filter combinations employed in the penumbra, as well as some of the umbra. It should be noted that there are also significant
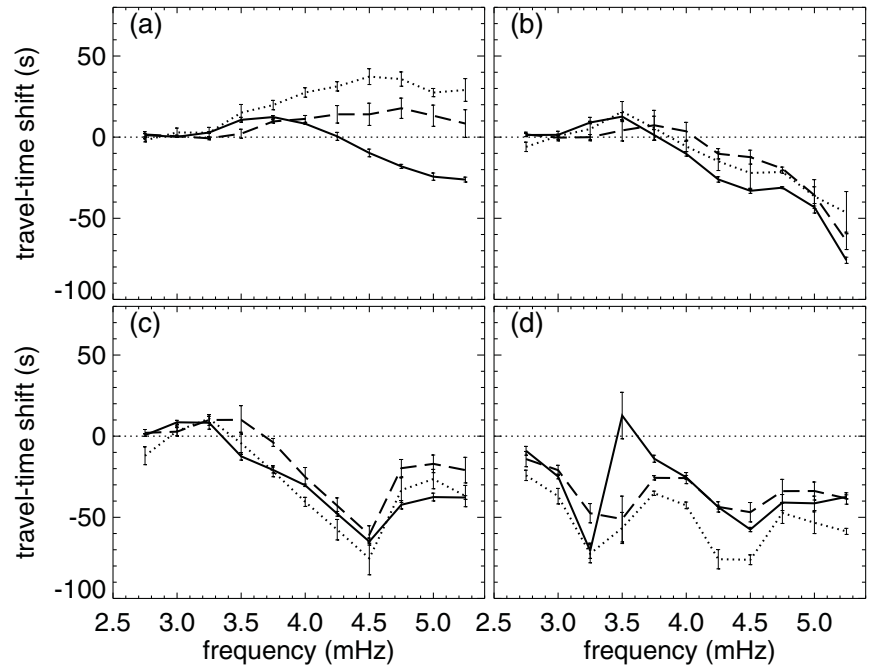

Figure 11. Averages over the umbra of the mean travel-time shifts for the simulation and the two sunspots with an amplitude modulation correction applied to the data (see the text). The lines have the same meaning as for Figure 9.

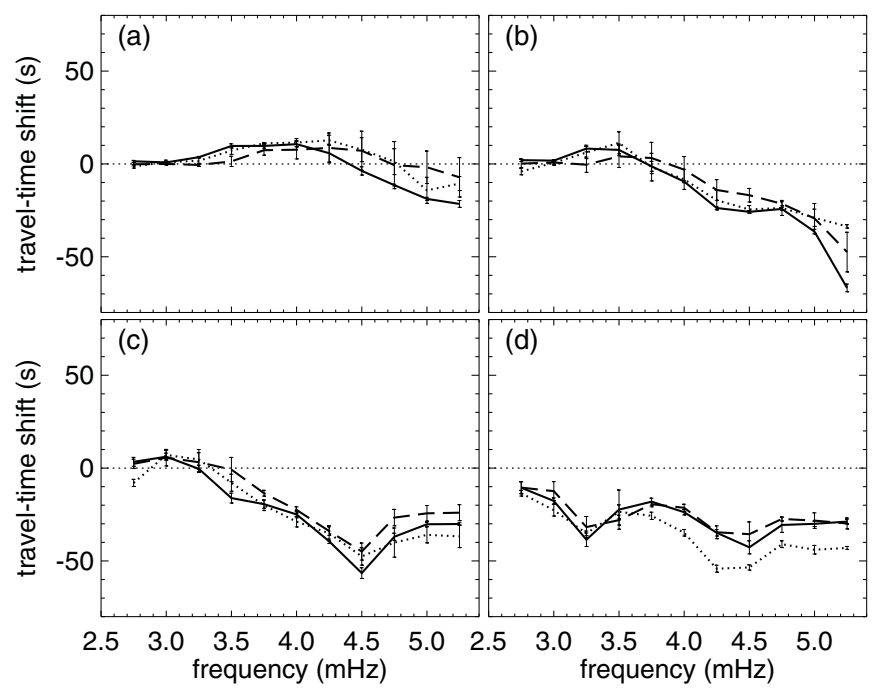

Figure 12. Averages over the penumbra of the mean travel-time shifts for the simulation and the two sunspots with an amplitude modulation correction applied to the data (see the text). The lines have the same meaning as for Figure 9.

differences, for some filter combinations, between the two real sunspots. These travel-time differences are comparable to those observed between the simulated spot and either of the real spots. Thus, we consider the overall agreement between the $\mathrm{HH}$ traveltime shifts observed in the simulated spot and those observed in real sunspots to be highly significant.

In some travel-time measurements applied to active region observations (e.g., Gizon et al. 2009; Zharkov et al. 2011) an amplitude modulation correction (Rajaguru et al. 2006) is applied to the data before filtering. The purpose of this is to account for the possibility that the spatial variation of wave amplitude in magnetic regions can produce systematic artifacts in travel times (Rajaguru et al. 2006; Parchevsky et al. 2008). Although such a correction is not universally accepted (Nigam \& Kosovichev 2010), we follow the lead of prior studies (e.g., Couvidat \& Rajaguru 2007; Braun \& Birch 2008) which assessed the possible impact of amplitude variations by performing the analysis both with and without an amplitude modulation. Figures 11 and 12 show the umbral and penumbral 

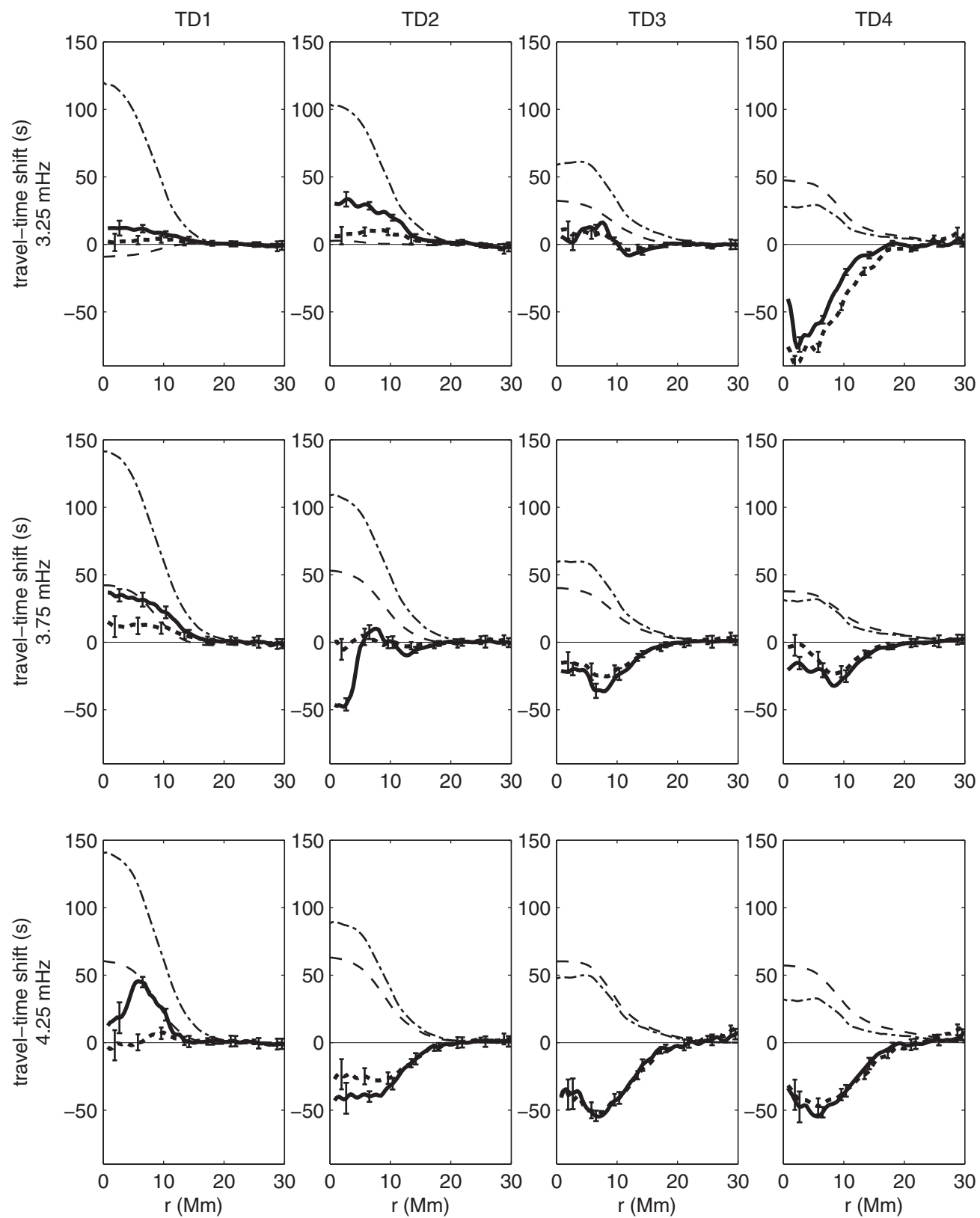

Figure 13. Comparisons of measured $\mathrm{HH}$ travel-time shifts from the model sunspot and expected travel-time shifts computed from the known sound-speed perturbation associated with the model. The solid (short-dashed) lines with error bars show the measured travel-time shifts without (with) the amplitude modulation correction (see the text). The other two lines (without error bars) show the results computed in the Born approximation (dashed lines) and the ray approximation (dot-dashed lines) from the sound-speed perturbation in the model sunspot. Each panel shows the azimuthal averages of the travel-time shifts as functions of distance from the center of the sunspot for a particular combination of phase-speed filter (TD1-TD4) and frequency filter $(3.25,3.75$, and $4.25 \mathrm{mHz})$.

averages, respectively, for the simulated and real sunspots from an analysis of the data after multiplying each pixel by the reciprocal of the square root of the wave power integrated over each frequency bandpass. For both real and simulated spots, the primary result of the correction procedure is a decrease of the positive travel-time shifts observed in filters TD1 and TD2 compared to the uncorrected travel-time shifts (Figures 9 and 10). Qualitatively, the good agreement between the results for simulated and real spots remains.

\section{FORWARD MODELING RESULTS}

In TD inversions, travel-time shifts have commonly been formally represented as resulting from changes in the local sound speed (e.g., Kosovichev \& Duvall 1997; Kosovichev et al. 2000; Hughes et al. 2005). This change in the local sound speed has been interpreted as a proxy for all physical effects that change wave speeds (e.g., temperature or magnetic field; Kosovichev et al. 2000).

Here we test the simple assumption that the observed traveltime shifts can be explained as resulting from the actual perturbation to the sound speed in the simulated sunspot. Traditional time-distance inversions assume a linear relationship between the perturbation to the sound speed (or square of the sound speed) and the observed travel-time shifts,

$$
\delta \tau=\iiint_{\odot} K(\boldsymbol{x}) \delta c^{2}(\boldsymbol{x}) / c_{0}^{2}(z) d \boldsymbol{x},
$$

where $K$ is a sensitivity (kernel) function, $c_{0}(z)$ is the quiet-Sun sound speed, and $\delta c^{2}=c^{2}-c_{0}^{2}$ is the perturbation to the square of the sound speed, $\boldsymbol{x}$ is a position vector, and $z$ is depth. We have 
computed the kernel functions both in the Born approximation (Birch et al. 2011; these kernels take into account the holography Green's functions, the phase-speed and frequency bandpass filters applied in the data analysis, the pupil sizes, and are based on a linearization of the phase method of measuring travel-time shifts) and the ray approximation (Kosovichev \& Duvall 1997). In the ray approximation, we computed the acoustic cutoff frequency using $\omega_{\mathrm{ac}}^{2}=c^{2} / 4 H^{2}(1-2 d H / d z)$ with $z$ increasing upward and $H$ is the density scale height (e.g., Equation (7.89) of Christensen-Dalsgaard 2003).

In Figure 13 we compare the travel-time shifts measured from the simulation (with and without the amplitude modulation correction) with the predictions of Equation (2) using both the Born and ray approximations. The figure shows the azimuthal averages of the travel-time shifts as functions of distance from the center of the sunspot for combinations of phase-speed and frequency filters, with the frequency filters centered at 3.25, 3.75, and $4.25 \mathrm{mHz}$. For some filter combinations (e.g., TD1 at $3.25 \mathrm{mHz}$ ) there are very large differences between the ray and Born approximations; these occur where the filtered power is dominated by non-ridge power. In general, neither the ray nor Born approximations for the travel-time shifts caused by the known sound-speed perturbation are able to explain the measured travel-time shifts. This is true for measurements made with and without the amplitude modulation correction. Thus, we find that the simple assumption of Equation (2) is not able to explain the measured travel-time shifts.

As another test, we replaced the perturbation to the square of the sound speed in Equation (2) with the perturbation to the square of the fast-mode speed $\left(c_{f}^{2}=c^{2}+a^{2}\right.$ where $a$ is the Alfvén speed). The fractional perturbation to the fastmode speed is much larger than one in much of the simulated sunspot (see Figure 2). The formal computation of Equation (2) in this case yields travel-time shifts of order 10 minutes, in contradiction to the observed travel times. We note, however, that the derivation of Equation (2) requires $\delta c^{2} / c_{0}^{2}$ to be small, which is certainly not the case when $c$ is taken to represent the fast-mode speed.

\section{DISCUSSION}

Attempts to model the travel times measured from this realistic MHD simulation have shown that Equation (2), or a simple modification thereof that accounts for the fast-mode speed, is not sufficient for interpreting either time-distance or $\mathrm{HH}$ measurements. This result is in marked contrast to a recent analysis of simulated data containing only pure sound-speed perturbations (Birch et al. 2011) where there was generally qualitative agreement between the measured and predicted travel-time shifts using sensitivity kernels computed under the assumption of the Born approximation.

Insofar as the measurements made with this simulation approximately match those found in typical sunspots, the simulation provides a reasonable model of the wave propagation through those spots. We note that the strong, near-surface, wave-speed perturbations of this model (see Figure 2) are in general qualitative agreement with other (forward) models of wave propagation in sunspot-like magnetic fields (Crouch et al. 2005; Cameron et al. 2011). The similarities between the traveltime measurements made using the artificial sunspot and actual observations of sunspots suggest that the simulation is sufficiently "realistic" to reproduce most of the relevant helioseismic properties of real sunspots. Developing an inversion method which can account for the complicated influences of the strong magnetic fields on travel-time shifts, while apparently necessary, remains a challenge for sunspot seismology. Some promise is shown by the development of inversion kernels which explicitly treat the effects of magnetic fields as perturbations about a magnetostatic reference model (e.g., Crouch et al. 2011; Hanasoge et al. 2011). It is not currently clear what other influences may need to be addressed. For example, it may also be useful to account for changes in density (e.g., the Wilson depression; Lindsey et al. 2010).

This work is supported by the NASA SDO Science Center program through contract NNH09CE41C awarded to NWRA, grant NNH09AK021 awarded to NCAR, and grant SCEX22011D awarded to NASA GSFC. Additional support is provided by the NASA Heliophysics GI program through contract NNG07EI51C awarded to NWRA and a subcontract through the NASA sponsored HMI project at Stanford University awarded to NWRA. The National Center for Atmospheric Research is sponsored by the National Science Foundation. $\mathrm{SOHO}$ is a project of international cooperation between ESA and NASA. SDO data are provided courtesy of NASA/SDO and the AIA, EVE, and HMI science teams. The authors acknowledge the Texas Advanced Computing Center (TACC) at The University of Texas at Austin for providing HPC resources that have contributed to the research results reported within this paper. We thank the referee for useful comments which helped to improve the manuscript.

\section{REFERENCES}

Basu, S., Antia, H. M., \& Bogart, R. S. 2004, ApJ, 610, 1157

Benson, D., Stein, R., \& Nordlund, A. 2006, in ASP Conf. Ser. 354, Solar MHD Theory and Observations: A High Spatial Resolution Perspective, ed. J. Leibacher, R. F. Stein, \& H. Uitenbroek (San Francisco, CA: ASP), 92

Birch, A. C., Braun, D. C., Hanasoge, S. M., \& Cameron, R. 2009, Sol. Phys., 254, 17

Birch, A. C., Kosovichev, A. G., Price, G. H., \& Schlottmann, R. B. 2001, ApJ, 561, L229

Birch, A. C., Parchevsky, K., Braun, D. C., \& Kosovichev, A. 2011, Sol. Phys., 272,11

Bogdan, T. J., \& Braun, D. C. 1995, in Helioseismology, Vol. 376, ed. J. T. Hoeksema, V. Domingo, B. Fleck, \& B. Battrick (Noordwijk: ESA), 31

Braun, D. C. 1995 , ApJ, 451, 859

Braun, D. C., \& Birch, A. C. 2006, ApJ, 647, L187

Braun, D. C., \& Birch, A. C. 2008, Sol. Phys., 251, 267

Braun, D. C., Birch, A. C., Benson, D., Stein, R. F., \& Nordlund, A. 2007, ApJ, 669,1395

Braun, D. C., Birch, A. C., Crouch, A., \& Rempel, M. 2011, J. Phys.: Conf. Ser., 271, 012010

Cameron, R., Gizon, L., \& Duvall, T., Jr. 2008, Sol. Phys., 251, 291

Cameron, R. H., Gizon, L., Schunker, H., \& Pietarila, A. 2011, Sol. Phys., 268, 293

Christensen-Dalsgaard, J. 2003, Lecture Notes on Stellar Oscillations (5th ed.; Institute for Fysik og Astronomi, Aarhus Universitet) (http://users-phys.au.dk/jcd/oscilnotes/)

Couvidat, S., Birch, A. C., \& Kosovichev, A. G. 2006, ApJ, 640, 516

Couvidat, S., Gizon, L., Birch, A. C., Larsen, R. M., \& Kosovichev, A. G. 2005, ApJS, 158, 217

Couvidat, S., \& Rajaguru, S. P. 2007, ApJ, 661, 558

Crouch, A. D., Birch, A. C., Braun, D. C., \& Clack, C. T. M. 2011, in IAU. Symp. 273, Physics of Sun and Star Spots, ed. D. P. Choudhary \& K. G. Strassmeier (Cambridge: Cambridge Univ. Press), 384

Crouch, A. D., Cally, P. S., Charbonneau, P., Braun, D. C., \& Desjardins, M. 2005, MNRAS, 363, 1188

Duvall, T. L., Jr., Kosovichev, A. G., Scherrer, P. H., et al. 1997, Sol. Phys., 170, 63

Fan, Y., Braun, D. C., \& Chou, D.-Y. 1995, ApJ, 451, 877

Gizon, L., \& Birch, A. C. 2002, ApJ, 571, 966

Gizon, L., \& Birch, A. C. 2005, Living Rev. Sol. Phys. (http://solarphysics. livingreviews.org/Articles/lrsp-2005-6/)

Gizon, L., Birch, A. C., \& Spruit, H. C. 2010, ARA\&A, 48, 289 
Gizon, L., Schunker, H., Baldner, C. S., et al. 2009, Space Sci. Rev., 144, 249

Hanasoge, S. M., Birch, A., Gizon, L., \& Tromp, J. 2011, ApJ, 738, 100

Hanasoge, S. M., Larsen, R. M., Duvall, T. L., Jr., et al. 2006, ApJ, 648, 1268

Hindman, B. W., Gizon, L., Duvall, T. L., Jr., Haber, D. A., \& Toomre, J. 2004, ApJ, 613, 1253

Hughes, S. J., Rajaguru, S. P., \& Thompson, M. J. 2005, ApJ, 627, 1040

Jensen, J. M., Olsen, K. B., Duvall, T. L., Jr., \& Jacobsen, B. H. 2003, in GONG+ 2002, Local and Global Helioseismology: The Present and Future, Vol. 517, ed. H. Sawaya-Lacoste (Noordwijk: ESA), 319

Khomenko, E., \& Collados, M. 2006, ApJ, 653, 739

Korzennik, S. G. 2006, in Proc. SOHO 18/GONG 2006/HELAS I, Beyond the Spherical Sun, Vol. 624, ed. K. Fletcher, Sci. ed., M. Thompson (Noordwijk: ESA)

Kosovichev, A. G. 1996, ApJ, 461, L55

Kosovichev, A. G. 2010, arXiv:1010.4929

Kosovichev, A. G., Basu, S., Bogart, R., et al. 2011, J. Phys.: Conf. Ser., 271, 012005

Kosovichev, A. G., \& Duvall, T. L., Jr. 1997, in SCORe'96: Solar Convection and Oscillations and their Relationship, ed. F. P. Pijpers, J. ChristensenDalsgaard, \& C. S. Rosenthal (Astrophysics and Space Science Library, Vol. 225; Dordrecht: Kluwer), 241

Kosovichev, A. G., Duvall, T. L., Jr., \& Scherrer, P. H. 2000, Sol. Phys., 192, 159

Lindsey, C., \& Braun, D. C. 2000, Sol. Phys., 192, 261

Lindsey, C., \& Braun, D. C. 2005, ApJ, 620, 1118

Lindsey, C., Cally, P. S., \& Rempel, M. 2010, ApJ, 719, 1144

Mansour, N. N., Kosovichev, A. G., Georgobiani, D., Wray, A., \& Miesch, M. 2004, in SOHO 14 Helio- and Asteroseismology: Towards a Golden Future, Vol. 559, ed. D. Danesy (Noordwijk: ESA), 164
Moradi, H., Baldner, C., Birch, A. C., et al. 2010, Sol. Phys., 267,

Moradi, H., Hanasoge, S. M., \& Cally, P. S. 2009, ApJ, 690, L72

Nigam, R., \& Kosovichev, A. G. 2010, ApJ, 708, 1475

Parchevsky, K. V., \& Kosovichev, A. G. 2007, ApJ, 666, 547

Parchevsky, K. V., \& Kosovichev, A. G. 2009, ApJ, 694, 573

Parchevsky, K. V., Zhao, J., \& Kosovichev, A. G. 2008, ApJ, 678, 1498

Rajaguru, S. P., Birch, A. C., Duvall, T. L., Jr., Thompson, M. J., \& Zhao, J. 2006, ApJ, 646, 543

Rempel, M. (ed.) 2010, IAU Symp. 6, International Meteor Conference, Bereges, France, 2008 June 7-10 (Cambridge: Cambridge Univ. Press), 8

Rempel, M. 2011, ApJ, 740, 15

Rempel, M., Schüssler, M., Cameron, R. H., \& Knölker, M. 2009a, Science, 325,171

Rempel, M., Schüssler, M., \& Knölker, M. 2009b, ApJ, 691, 640

Scherrer, P. H., Bogart, R. S., Bush, R. I., et al. 1995, Sol. Phys., 162, 129

Schou, J., Scherrer, P. H., Bush, R. I., Wachter, R., \& Couvidat, S. 2011, Sol. Phys. (http://www.springerlink.com/content/n0k3765711577156/)

Schunker, H., Braun, D. C., \& Cally, P. S. 2007, Astron. Nachr., 328, 292

Schunker, H., Braun, D. C., Cally, P. S., \& Lindsey, C. 2005, ApJ, 621, L149

Schunker, H., Braun, D. C., Lindsey, C., \& Cally, P. S. 2008, Sol. Phys., 251, 341

Shelyag, S., Erdélyi, R., \& Thompson, M. J. 2006, ApJ, 651, 576

Shelyag, S., Erdélyi, R., \& Thompson, M. J. 2007, A\&A, 469, 1101

Thompson, M. J., \& Zharkov, S. 2008, Sol. Phys., 251, 225

Tong, C. H., Thompson, M. J., Warner, M. R., \& Pain, C. C. 2003, ApJ, 593, 1242

Zhao, J., Georgobiani, D., Kosovichev, A. G., et al. 2007, ApJ, 659, 848

Zhao, J., \& Kosovichev, A. G. 2006, ApJ, 643, 1317

Zhao, J., Kosovichev, A. G., \& Sekii, T. 2010, ApJ, 708, 304

Zharkov, S., Zharkova, V. V., \& Matthews, S. A. 2011, ApJ, 739, 70 